\title{
A note on perfect Lucky k-colourable graphs
}

\author{
Johan Kok ${ }^{\mathrm{a}}$, Joseph Varghese Kureethara ${ }^{\mathrm{b}, *}$ \\ a Independent Mathematics Researcher, City of Tshwane, South Africa. \\ ${ }^{b}$ CHRIST (Deemed to be a University), Bangalore, India.
}

\begin{abstract}
This paper presents the notion of perfect Lucky k-colouring. Basic conditions for a perfect Lucky k-colourable graph are presented. Application thereof is then presented by obtaining the Lucky 4-polynomials for all connected graphs $\mathrm{G}$ on six vertices with ten edges. The chromatic number of these connected graphs is $\chi(G)=3$ or 4 . For $k=\max \{\chi(G): 3$ or 4$\}=4$, it is possible to find Lucky 4-polynomials for all graphs on six vertices and ten edges. The methodology improves substantially on the fundamental methodology such that, vertex partitions begin with Lucky partition forms immediately. Finally, further problems for research related to this study are presented.
\end{abstract}

Keywords: Perfect Lucky k-colouring, Lucky k-polynomial, Lucky $\chi$-polynomial.

2020 MSC: 05C15, 05C38, 05C75, 05C85.

(C)2020 All rights reserved.

\section{Introduction}

For general notation and concepts in graphs see [1] and [4]. Unless stated otherwise, all graphs are simple connected finite graphs. The set of vertices and the set of edges of a graph $G$ are denoted by $V(G)$ and $E(G)$, respectively. The number of vertices is called the order of $G$, say, $n$ and the number of edges is called the size of $\mathrm{G}$ denoted by $\varepsilon(\mathrm{G})$.

In a proper colouring of $\mathrm{G}$, all edges are good i.e., $\mathrm{u} v \Leftrightarrow \mathfrak{c}(\mathrm{u}) \neq \mathrm{c}(v)$. For any proper colouring $\varphi(\mathrm{G})$ of a graph $G$ the addition of all good edges, if any, is called the chromatic completion of $G$ in respect of $\varphi(\mathrm{G})$. The additional edges are called chromatic completion edges. The set of such chromatic completion edges is denoted by $E_{\varphi}(G)$. The resultant graph $G_{\varphi}$ is called a chromatic completion graph of $G$.

The chromatic completion number of a graph $G$ denoted by $\zeta(G)$ is the maximum number of good edges that can be added to $G$ over all chromatic colourings ( $\chi$-colourings). Hence, $\zeta(G)=\max \left\{\left|E_{\chi}(G)\right|\right.$ : over all $\left.\varphi_{\chi}(\mathrm{G})\right\}$.

\footnotetext{
${ }^{*}$ Corresponding author

Email addresses: jacotype@gmail.com (Johan Kok), frjoseph@christuniversity.in (Joseph Varghese Kureethara)

doi: $10.22436 /$ jmcs.021.03.03
}

Received: 2019-09-26 Revised: 2020-02-17 Accepted: 2020-03-13 


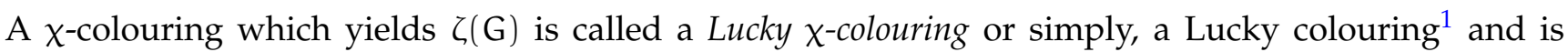
denoted by $\varphi_{\mathcal{L}}(\mathrm{G})$. The resultant graph $\mathrm{G}_{\zeta}$ is called a minimal chromatic completion graph of $\mathrm{G}$. It is trivially true that $\mathrm{G} \subseteq \mathrm{G}_{\zeta}$. Furthermore, the graph induced by the set of completion edges, $\left\langle\mathrm{E}_{\chi}\right\rangle$ is a subgraph of the complement graph $\overline{\mathrm{G}}$.

A k-colouring of a graph $G$ which yields $\max \left\{\left|\mathrm{E}_{\varphi}(\mathrm{G})\right|:\right.$ overall k-colourings $\}$ is called a Lucky kcolouring.

In an improper colouring an edge $u v$ for which, $c(u)=c(v)$ is called a bad edge. It is observed that the number of edges of $\bar{G}$ which are omitted from $E_{X}$ is the minimum number of bad edges in a bad chromatic completion of a graph G.

\section{Perfect Lucky Colourings}

If a graph is k-colourable in accordance with Lucky's theorem, then, in accordance with the Lucky partition form,

$$
\begin{aligned}
& \{\underbrace{\left\{\left\lfloor\frac{n}{k}\right\rfloor \text {-element }\right\},\left\{\left\lfloor\frac{n}{k}\right\rfloor \text {-element }\right\}, \ldots,\left\{\left\lfloor\frac{n}{k}\right\rfloor \text {-element }\right\}}_{(k-r) \text {-subsets }}, \\
& \underbrace{\left.\left\{\left\lceil\frac{\mathrm{n}}{\mathrm{k}}\right\rceil \text { - element }\right\},\left\{\left\lceil\frac{\mathrm{n}}{\mathrm{k}}\right\rceil \text { - element }\right\}, \ldots,\left\{\left\lceil\frac{\mathrm{n}}{\mathrm{k}}\right\rceil \text { - element }\right\}\right\}}_{(\mathrm{r} \geqslant 0) \text {-subsets }},
\end{aligned}
$$

$\mathrm{G}$ is said to be perfect Lucky k-colourable.[3]

Recall that the adjacency matrix of a graph $G$ of order $n$ is a $[n \times n]$-symmetrical $(0,1)$-matrix with zero's on the diagonal and entry $a_{i j}=1, i \neq j$ if and only if vertices $v_{i}, v_{j}$ are adjacent, else $a_{i j}=0$. Let the number of zero entries be denoted by $\mathfrak{O}_{\mathrm{G}}$. This brings the first trivial result.

Corollary 2.1. The coefficient of the Lucky k-polynomial of a graph $\mathrm{G}$ of order $k+1$ is, $\varepsilon(\overline{\mathrm{G}})$.

Proof. The Lucky partition form is given by:

$$
\{\{2-\text { element }\}, \underbrace{\{1-\text { element }\}, \ldots,\{1-\text { element }\}}_{(k-1) \text { subsets }}\} .
$$

Since an edge in $\bar{G}$ represents an independent $\{2$-element $\}$ vertex subset of $V(G)$, the result follows.

Corollary 2.1 can be put differently by considering the adjacency matrix of G. Consider the matrix 2.1 with $a_{i j}=0$ or 1 given below

$$
\left(\begin{array}{cccc}
0 & a_{12} & \ldots & a_{1 n} \\
a_{21} & 0 & \ldots & e_{2 n} \\
\ldots \ldots & \ldots \ldots \ldots \ldots \ldots . \\
a_{n 1} & a_{n 2} & \ldots & 0
\end{array}\right)
$$

Since a non-diagonal zero entry represents an independent $\{2$-element $\}$ subset of $V(G)$, the coefficient of the Lucky k-polynomial of a graph $G$ of order $k+1$ is $\frac{\mathfrak{O}_{G}-n}{2}$.

Hence, we have another useful result.

Corollary 2.2. For a graph of order $n \geqslant 2$, all connected graphs are $K_{n}$ and clusters of graphs with number of edges given by $\frac{\mathfrak{n}(\mathfrak{n}-1)}{2}-i, i=1,2,3, \ldots, \frac{(n-1)(n-2)}{2}$. For each graph in a cluster the coefficient of the perfect Lucky $(\mathrm{n}-1)$-colouring is $i$.

\footnotetext{
${ }^{1}$ Note that a Lucky colouring is an alias for an equitable $x$-colouring. The alias is meant to associate the paper with Lucky's Theorem and the notion of chromatic completion.
} 
Proof. Since $\varepsilon(\overline{\mathrm{G}})=i$ the result is immediate from Corollary 2.1 .

The coefficient of the Lucky k-polynomial of a graph $G$ of order $k+2$ can be determined by the following procedure.

Lemma 2.3. Let $\mathrm{G}$ be a simple connected graph with complement $\overline{\mathrm{G}}$, line graph $\mathrm{L}(\overline{\mathrm{G}})$ and adjacency matrix of the complement of the line graph $\mathrm{H}=\mathrm{L}(\overline{\mathrm{G}})$. Since the adjacency matrix is $a\left[\frac{\mathrm{n}(\mathrm{n}-1)}{2}-\varepsilon(\mathrm{G}) \times \frac{\mathrm{n}(\mathrm{n}-1)}{2}-\varepsilon(\mathrm{G})\right]-$ symmetrical $(0,1)$-matrix, the coefficient of the Lucky k-polynomial is $\frac{\left(\mathfrak{D}_{\mathrm{H}}-\left(\frac{\mathfrak{n}(\mathfrak{n}-1)}{2}-\varepsilon(\mathrm{G})\right)\right)}{2}$.

Proof. The Lucky partition form is given by:

$$
\{\{2-\text { element }\},\{2-\text { element }\}, \underbrace{1-\text { element }\}, \ldots,\{1-\text { element }\}}_{(\mathrm{k}-2) \text { subsets }}\} .
$$

Each edge in $\bar{G}$ represents an independent \{2-element $\}$ vertex subset of $V(G)$. However, in the prescribed vertex partition the two \{2-element $\}$ subsets may not have a common endpoint in G. Put differently, their intersection must be empty. Such a common endpoint is represented by an edge in the line graph $H=L(\bar{G})$. Therefore, a non-diagonal zero entry in the adjacency matrix of $H$ represents two independent \{2-element $\}$ subsets of $V(G)$. In the count of all zero entries in the adjacency matrix of $H$, the non-diagonal zero entries are subject to a double count and the diagonal zero's are irrelevant. Hence, the coefficient of the corresponding Lucky k-polynomial is $\frac{\left(\mathfrak{O}_{\mathrm{H}}-\left(\frac{\mathfrak{n}(\mathfrak{n}-1)}{2}-\varepsilon(\mathrm{G})\right)\right)}{2}$.

If two induced subgraphs of $G$ share no common vertex (vertices) it is said that the subgraphs are independent or 0-overlap subgraphs. For three or more, say, $t$ mutually independent induced subgraphs it is said to be $\mathrm{t}$-0-overlap subgraphs. For brevity, a clique $\mathrm{Q}$ of order $\mathrm{n}$ is called an $n$-clique and an independent set I of size $n$ is called an $n$-independent set. A result and a dual result on perfect Lucky k-colourability of a graph are presented next.

Theorem 2.4. Let graph $\mathrm{G}$ of order $\mathrm{n}=\mathrm{m} k+\mathrm{r}, \mathrm{m} \geqslant 2,2 \leqslant \mathrm{k} \leqslant \mathrm{n}-1$ and $1 \leqslant \mathrm{r} \leqslant \mathrm{k}$. A graph $\mathrm{G}$ is perfect Lucky $\mathrm{k}$-colourable if and only if $\overline{\mathrm{G}}$ has at least $\mathrm{r},(\mathrm{m}+1)$-cliques which are $\mathrm{r}$-0-overlap cliques and distinct from that, it has at least $(\mathrm{k}-\mathrm{r}), \mathrm{m}$-cliques which are $(\mathrm{k}-\mathrm{r})$-0-overlap cliques.

Proof. Obviously, any connected graph $\mathrm{G}$ of order $n \geqslant 2$ is not Lucky 1 -colourable and therefore, not perfect Lucky 1 -colourable. Also, any graph of order $n$ is perfect Lucky $n$-colourable. Thus we have the bounds $2 \leqslant k \leqslant n-1$.

If at least $r$ independent $K_{m+1}$ 's exist in $\bar{G}$, then at least $r(m+1)$-independent vertex subsets exist in G. Hence, at least $r\{(m+1)$-element $\}$ subsets in a corresponding Lucky partition form exist. If distinct from the aforesaid, at least $(k-r)$ independent $K_{m}$ 's exist in $\bar{G}$, then by similar reasoning it follows that at least $(k-r), m$-independent vertex subsets exist in $G$. It implies that at least one Lucky partition of the Lucky partition form given below exists

$$
\begin{gathered}
\{\underbrace{\{m-\text { element }\},\{m-\text { element }\}, \ldots,\{m-\text { element }\}}_{(k-r)-\text { subsets }}, \\
\underbrace{\{(m+1)-\text { element }\},\{(m+1)-\text { element }\}, \ldots,\{(m+1)-\text { element }\}}_{(r \geqslant 0)-\text { subsets }}\} .
\end{gathered}
$$

Through immediate induction it follows from similar reasoning that it holds $\forall k, 2 \leqslant k \leqslant n-1$. Therefore, $\mathrm{G}$ is perfect Lucky k-colourable, else not.

Conversely, if $\mathrm{G}$ is perfect $\mathrm{k}$-colourable then the definition of a proper colouring read with the definition of a perfect Lucky k-colouring unwraps the result.

Theorem 2.5. Let graph $\mathrm{G}$ of order $\mathrm{n}=\mathrm{mk}+\mathrm{r}, \mathrm{m} \geqslant 2,2 \leqslant \mathrm{k} \leqslant \mathrm{n}-1$ and $1 \leqslant \mathrm{r} \leqslant \mathrm{k}$. A graph $\mathrm{G}$ is perfect Lucky $\mathrm{k}$-colourable if and only if $\mathrm{G}$ has at least $\mathrm{r}(\mathrm{m}+1)$-independent sets which are pairwise disjoint and distinct from that, it has at least $(\mathrm{k}-\mathrm{r}) \mathrm{m}$-independent sets which are pairwise disjoint. 
Proof. The result follows by similar dual reasoning as in the proof of Theorem 2.4.

Recall the bounds for the chromatic number, $\chi(G)$, the clique number, $\omega(G)$ and the independence number, $\alpha(\mathrm{G})$

1. $1 \leqslant \chi(G) \leqslant n ;$

2. $\chi(G) \leqslant \omega(G)$;

3. $n \leqslant \chi(G) \alpha(G)$.

The third bound read together with Lemma 2.3 provides the following corollary.

Corollary 2.6. A graph $\mathrm{G}$ of order $\mathrm{mk}+1, \mathrm{~m} \geqslant 2$ is perfect Lucky $\mathrm{k}$-colourable if and only if $\mathrm{m}+1 \leqslant \alpha(\mathrm{G})$.

Proof. If $\mathrm{G}$ of order $\mathrm{mk}+1, \mathrm{~m} \geqslant 2$ is perfect Lucky $\mathrm{k}$-colourable then at least one independent $\{(\mathrm{m}+1)$ element $\}$ subset of $V(G)$ exists. Hence, $\alpha(G) \geqslant m+1$.

For $\alpha(G)$ at least one of each of the independent subsets of $V(G)$, say, $X_{i}, i=1,2,3, \ldots, \alpha(G)$ exist such that, $\left|X_{i}\right|=i$. Hence, if $\alpha(G) \geqslant m+1$, then at least one independent $\{(m+1)$-element $\}$ subset of $V(G)$ exists. Therefore, the result.

\section{Lucky 4-Polynomials of $\mathrm{G}$ of Order 6 and Size 10}

It is well-known that for a set of colours $\mathcal{C},|\mathcal{C}|=\lambda \geqslant \chi(G) \geqslant 2$, a graph $\mathrm{G}$ of order $n$ can always be coloured properly in $\mathcal{P}_{\mathrm{G}}(\lambda, n)$ distinct ways. The polynomial $\mathcal{P}_{\mathrm{G}}(\lambda, n)$ is called the chromatic polynomial of $\mathrm{G}$.

It is known that for $\chi(G) \leqslant n \leqslant \lambda$ colours, the number of distinct Lucky k-colourings, $\chi(G) \leqslant k \leqslant n$ is determined by a polynomial, called the Lucky k-polynomial, $\mathcal{L}_{\mathrm{G}}(\lambda, k)$.

In [2, pp. 38-40], all connected graphs of order 6 are depicted. In this paper, the graph numbering, $G_{i}, i=1,2,3, \ldots, 112$ in [2] as well as the clustering in equal size will be utilized. This section will relate to graphs of order 6 and size 10, i.e., graphs $G_{19}$ to $G_{32}$. The chromatic number of the respective graphs under study is either 3 or 4 . We study a Lucky 4-colouring throughout to study perfect Lucky 4 -colourings and to obtain Lucky 4-polynomials. Let the number of colours be $\lambda \geqslant 4$. The Lucky partition form is:

\section{$\{\{2$-element $\},\{2$-element $\},\{1$-element $\},\{1$-element $\}\}$.}

Hence, the corresponding Lucky partitions of $\mathrm{V}(\mathrm{G})$ are:

$\left\{\left\{v_{1}, v_{2}\right\},\left\{v_{3}, v_{4}\right\},\left\{v_{5}\right\},\left\{v_{6}\right\}\right\},\left\{\left\{v_{1}, v_{2}\right\},\left\{v_{3}, v_{5}\right\},\left\{v_{4}\right\},\left\{v_{6}\right\}\right\},\left\{\left\{v_{1}, v_{2}\right\},\left\{v_{3}\right\},\left\{v_{4}, v_{5}\right\},\left\{v_{6}\right\}\right\},\left\{\left\{v_{1}, v_{2}\right\},\left\{v_{3}, v_{6}\right\},\left\{v_{4}\right\},\left\{v_{5}\right\}\right\}$, $\left\{\left\{v_{1}, v_{2}\right\},\left\{v_{3}\right\},\left\{v_{4}, v_{6}\right\},\left\{v_{5}\right\}\right\},\left\{\left\{v_{1}, v_{2}\right\},\left\{v_{3}\right\},\left\{v_{4}\right\},\left\{v_{5}, v_{6}\right\}\right\},\left\{\left\{v_{1}, v_{3}\right\},\left\{v_{2}, v_{4}\right\},\left\{v_{5}\right\},\left\{v_{6}\right\}\right\},\left\{\left\{v_{1}, v_{3}\right\},\left\{v_{2}, v_{5}\right\},\left\{v_{4}\right\},\left\{v_{6}\right\}\right\}$, $\left\{\left\{v_{1}, v_{3}\right\},\left\{v_{2}\right\},\left\{v_{4}, v_{5}\right\},\left\{v_{6}\right\}\right\},\left\{\left\{v_{1}, v_{3}\right\},\left\{v_{2}, v_{6}\right\},\left\{v_{4}\right\},\left\{v_{5}\right\}\right\},\left\{\left\{v_{1}, v_{3}\right\},\left\{v_{2}\right\},\left\{v_{4}, v_{6}\right\},\left\{v_{5}\right\}\right\},\left\{\left\{v_{1}, v_{3}\right\},\left\{v_{2}\right\},\left\{v_{4}\right\},\left\{v_{5}, v_{6}\right\}\right\}$, $\left\{\left\{v_{1}, v_{4}\right\},\left\{v_{2}, v_{3}\right\},\left\{v_{5}\right\},\left\{v_{6}\right\}\right\},\left\{\left\{v_{1}, v_{5}\right\},\left\{v_{2}, v_{3}\right\},\left\{v_{4}\right\},\left\{v_{6}\right\}\right\},\left\{\left\{v_{1}\right\},\left\{v_{2}, v_{3}\right\},\left\{v_{4}, v_{5}\right\},\left\{v_{6}\right\}\right\},\left\{\left\{v_{1}, v_{6}\right\},\left\{v_{2}, v_{3}\right\},\left\{v_{4}\right\},\left\{v_{5}\right\}\right\}$, $\left\{\left\{v_{1}\right\},\left\{v_{2}, v_{3}\right\},\left\{v_{4}, v_{6}\right\},\left\{v_{5}\right\}\right\},\left\{\left\{v_{1}\right\},\left\{v_{2}, v_{3}\right\},\left\{v_{4}\right\},\left\{v_{5}, v_{6}\right\}\right\},\left\{\left\{v_{1}, v_{4}\right\},\left\{v_{2}, v_{5}\right\},\left\{v_{3}\right\},\left\{v_{6}\right\}\right\},\left\{\left\{v_{1}, v_{4}\right\},\left\{v_{2}\right\},\left\{v_{3}, v_{5}\right\},\left\{v_{6}\right\}\right\}$, $\left\{\left\{v_{1}, v_{4}\right\},\left\{v_{2}, v_{6}\right\},\left\{v_{3}\right\},\left\{v_{5}\right\}\right\},\left\{\left\{v_{1}, v_{4}\right\},\left\{v_{2}\right\},\left\{v_{3}, v_{6}\right\},\left\{v_{5}\right\}\right\},\left\{\left\{v_{1}, v_{4}\right\},\left\{v_{2}\right\},\left\{v_{3}\right\},\left\{v_{5}, v_{6}\right\}\right\},\left\{\left\{v_{1}, v_{5}\right\},\left\{v_{2}, v_{4}\right\},\left\{v_{3}\right\},\left\{v_{6}\right\}\right\}$, $\left\{\left\{v_{1}\right\},\left\{v_{2}, v_{4}\right\},\left\{v_{3}, v_{5}\right\},\left\{v_{6}\right\}\right\},\left\{\left\{v_{1}, v_{6}\right\},\left\{v_{2}, v_{4}\right\},\left\{v_{3}\right\},\left\{v_{5}\right\}\right\},\left\{\left\{v_{1}\right\},\left\{v_{2}, v_{4}\right\},\left\{v_{3}, v_{6}\right\},\left\{v_{5}\right\}\right\},\left\{\left\{v_{1}\right\},\left\{v_{2}, v_{4}\right\},\left\{v_{3}\right\},\left\{v_{5}, v_{6}\right\}\right\}$, $\left\{\left\{v_{1}, v_{5}\right\},\left\{v_{2}\right\},\left\{v_{3}, v_{4}\right\},\left\{v_{6}\right\}\right\},\left\{\left\{v_{1}\right\},\left\{v_{2}, v_{5}\right\},\left\{v_{3}, v_{4}\right\},\left\{v_{6}\right\}\right\},\left\{\left\{v_{1}, v_{6}\right\},\left\{v_{2}\right\},\left\{v_{3}, v_{4}\right\},\left\{v_{5}\right\}\right\},\left\{\left\{v_{1}\right\},\left\{v_{2}, v_{6}\right\},\left\{v_{3}, v_{4}\right\},\left\{v_{5}\right\}\right\}$, $\left\{\left\{v_{1}\right\},\left\{v_{2}\right\},\left\{v_{3}, v_{4}\right\},\left\{v_{5}, v_{6}\right\}\right\},\left\{\left\{v_{1}, v_{5}\right\},\left\{v_{2}, v_{6}\right\},\left\{v_{3}\right\},\left\{v_{4}\right\}\right\},\left\{\left\{v_{1}, v_{5}\right\},\left\{v_{2}\right\},\left\{v_{3}, v_{6}\right\},\left\{v_{4}\right\}\right\},\left\{\left\{v_{1}, v_{5}\right\},\left\{v_{2}\right\},\left\{v_{3}\right\},\left\{v_{4}, v_{6}\right\}\right\}$, $\left\{\left\{v_{1}, v_{6}\right\},\left\{v_{2}, v_{5}\right\},\left\{v_{3}\right\},\left\{v_{4}\right\}\right\},\left\{\left\{v_{1}\right\},\left\{v_{2}, v_{5}\right\},\left\{v_{3}, v_{6}\right\},\left\{v_{4}\right\}\right\},\left\{\left\{v_{1}\right\},\left\{v_{2}, v_{5}\right\},\left\{v_{3}\right\},\left\{v_{4}, v_{6}\right\}\right\},\left\{\left\{v_{1}, v_{6}\right\},\left\{v_{2}\right\},\left\{v_{3}, v_{5}\right\},\left\{v_{4}\right\}\right\}$, $\left\{\left\{v_{1}\right\},\left\{v_{2}, v_{6}\right\},\left\{v_{3}, v_{5}\right\},\left\{v_{4}\right\}\right\},\left\{\left\{v_{1}\right\},\left\{v_{2}\right\},\left\{v_{3}, v_{5}\right\},\left\{v_{4}, v_{6}\right\}\right\},\left\{\left\{v_{1}, v_{6}\right\},\left\{v_{2}\right\},\left\{v_{3}\right\},\left\{v_{4}, v_{5}\right\}\right\},\left\{\left\{v_{1}\right\},\left\{v_{2}, v_{6}\right\},\left\{v_{3}\right\},\left\{v_{4}, v_{5}\right\}\right\}$, $\left\{\left\{v_{1}\right\},\left\{v_{2}\right\},\left\{v_{3}, v_{6}\right\},\left\{v_{4}, v_{5}\right\}\right\}$.

For a graph $\mathrm{G}_{i}$, the vertex labeling will, by informal convention, be: "'Sweep a graph with a vertical line from left to right. If exactly one vertex is the first vertex that intersects, label it as $v_{1}$. If two or more vertices intersect simultaneously, then label consecutively from top to bottom say, $v_{1}, v_{2}, \ldots, v_{j}$ then proceed in similar fashion when the next intersecting vertex (or vertices), yields (or yield)."'

For a graph $G_{i}$, the permissible Lucky partitions are those in which adjacent vertices are not elements of a subset of a Lucky partition. Put differently, all subsets of a Lucky partition are independent sets. 


\subsection{Graphs $\mathrm{G}_{i}$ for which, $\chi\left(\mathrm{G}_{i}\right)=3$}

These graphs are $\mathrm{G}_{25}, \mathrm{G}_{27}, \mathrm{G}_{29}, \mathrm{G}_{30}, \mathrm{G}_{31}$ and $\mathrm{G}_{32}$.

(a) For graph $\mathrm{G}_{25}$, permissible Lucky partitions are,

$\left\{\left\{v_{1}, v_{4}\right\},\left\{v_{2}\right\},\left\{v_{3}, v_{5}\right\},\left\{v_{6}\right\}\right\},\left\{\left\{v_{1}, v_{5}\right\},\left\{v_{2}\right\},\left\{v_{3}\right\},\left\{v_{4}, v_{6}\right\}\right\},\left\{\left\{v_{1}, v_{6}\right\},\left\{v_{2}\right\},\left\{v_{3}, v_{5}\right\},\left\{v_{4}\right\}\right\},\left\{\left\{v_{1}\right\},\left\{v_{2}\right\},\left\{v_{3}, v_{5}\right\},\left\{v_{4}, v_{6}\right\}\right\}$. Therefore, $\mathrm{L}_{\mathrm{G}_{25}}(6,4)=4 \lambda(\lambda-1)(\lambda-2)(\lambda-3)$.

(b) For graph $\mathrm{G}_{27}$, permissible Lucky partitions are,

$\left\{\left\{v_{1}, v_{5}\right\},\left\{v_{2}, v_{4}\right\},\left\{v_{3}\right\},\left\{v_{6}\right\}\right\},\left\{\left\{v_{1}, v_{6}\right\},\left\{v_{2}, v_{4}\right\},\left\{v_{3}\right\},\left\{v_{5}\right\}\right\},\left\{\left\{v_{1}\right\},\left\{v_{2}, v_{4}\right\},\left\{v_{3}, v_{6}\right\},\left\{v_{5}\right\}\right\},\left\{\left\{v_{1}, v_{5}\right\},\left\{v_{2}, v_{6}\right\},\left\{v_{3}\right\},\left\{v_{4}\right\}\right\}$, $\left\{\left\{v_{1}, v_{5}\right\},\left\{v_{2}\right\},\left\{v_{3}, v_{6}\right\},\left\{v_{4}\right\}\right\}$.

Therefore, $\mathrm{L}_{\mathrm{G}_{27}}(6,4)=5 \lambda(\lambda-1)(\lambda-2)(\lambda-3)$.

(c) For graph $\mathrm{G}_{29}$, permissible Lucky partitions are,

$\left\{\left\{v_{1}, v_{5}\right\},\left\{v_{2}, v_{4}\right\},\left\{v_{3}\right\},\left\{v_{6}\right\}\right\},\left\{\left\{v_{1}, v_{6}\right\},\left\{v_{2}, v_{4}\right\},\left\{v_{3}\right\},\left\{v_{5}\right\}\right\},\left\{\left\{v_{1}\right\},\left\{v_{2}, v_{4}\right\},\left\{v_{3}, v_{6}\right\},\left\{v_{5}\right\}\right\},\left\{\left\{v_{1}\right\},\left\{v_{2}, v_{4}\right\},\left\{v_{3}\right\},\left\{v_{5}, v_{6}\right\}\right\}$, $\left\{\left\{v_{1}, v_{5}\right\},\left\{v_{2}\right\},\left\{v_{3}, v_{6}\right\},\left\{v_{4}\right\}\right\}$.

Therefore, $\mathrm{L}_{\mathrm{G}_{29}}(6,4)=5 \lambda(\lambda-1)(\lambda-2)(\lambda-3)$.

(d) For graph $\mathrm{G}_{30}$, permissible Lucky partitions are,

$\left\{\left\{v_{1}, v_{4}\right\},\left\{v_{2}, v_{5}\right\},\left\{v_{3}\right\},\left\{v_{6}\right\}\right\},\left\{\left\{v_{1}, v_{4}\right\},\left\{v_{2}, v_{6}\right\},\left\{v_{3}\right\},\left\{v_{5}\right\}\right\},\left\{\left\{v_{1}\right\},\left\{v_{2}, v_{5}\right\},\left\{v_{3}, v_{4}\right\},\left\{v_{6}\right\}\right\},\left\{\left\{v_{1}, v_{6}\right\},\left\{v_{2}\right\},\left\{v_{3}, v_{4}\right\},\left\{v_{5}\right\}\right\}$, $\left\{\left\{v_{1}\right\},\left\{v_{2}, v_{6}\right\},\left\{v_{3}, v_{4}\right\},\left\{v_{5}\right\}\right\},\left\{\left\{v_{1}, v_{6}\right\},\left\{v_{2}, v_{5}\right\},\left\{v_{3}\right\},\left\{v_{4}\right\}\right\}$.

Therefore, $\mathrm{L}_{\mathrm{G}_{30}}(6,4)=6 \lambda(\lambda-1)(\lambda-2)(\lambda-3)$.

(e) For graph $\mathrm{G}_{31}$, permissible Lucky partitions are,

$\left\{\left\{v_{1}, v_{5}\right\},\left\{v_{2}\right\},\left\{v_{3}, v_{4}\right\},\left\{v_{6}\right\}\right\},\left\{\left\{v_{1}\right\},\left\{v_{2}, v_{5}\right\},\left\{v_{3}, v_{4}\right\},\left\{v_{6}\right\}\right\},\left\{\left\{v_{1}, v_{6}\right\},\left\{v_{2}\right\},\left\{v_{3}, v_{4}\right\},\left\{v_{5}\right\}\right\},\left\{\left\{v_{1}\right\},\left\{v_{2}, v_{6}\right\},\left\{v_{3}, v_{4}\right\},\left\{v_{5}\right\}\right\}$, $\left\{\left\{v_{1}, v_{5}\right\},\left\{v_{2}, v_{6}\right\},\left\{v_{3}\right\},\left\{v_{4}\right\}\right\},\left\{\left\{v_{1}, v_{6}\right\},\left\{v_{2}, v_{5}\right\},\left\{v_{3}\right\},\left\{v_{4}\right\}\right\}$.

Therefore, $\mathrm{L}_{\mathrm{G}_{31}}(6,4)=6 \lambda(\lambda-1)(\lambda-2)(\lambda-3)$.

(f) For graph $\mathrm{G}_{32}$, permissible Lucky partitions are,

$\left\{\left\{v_{1}, v_{4}\right\},\left\{v_{2}, v_{3}\right\},\left\{v_{5}\right\},\left\{v_{6}\right\}\right\},\left\{\left\{v_{1}, v_{5}\right\},\left\{v_{2}, v_{3}\right\},\left\{v_{4}\right\},\left\{v_{6}\right\}\right\},\left\{\left\{v_{1}\right\},\left\{v_{2}, v_{3}\right\},\left\{v_{4}, v_{5}\right\},\left\{v_{6}\right\}\right\},\left\{\left\{v_{1}, v_{4}\right\},\left\{v_{2}\right\},\left\{v_{3}, v_{6}\right\},\left\{v_{5}\right\}\right\}$, $\left\{\left\{v_{1}, v_{5}\right\},\left\{v_{2}\right\},\left\{v_{3}, v_{6}\right\},\left\{v_{4}\right\}\right\}$.

Therefore, $\mathrm{L}_{\mathrm{G}_{32}}(6,4)=5 \lambda(\lambda-1)(\lambda-2)(\lambda-3)$.

Corollary 3.1. All graphs of order 6 and size 10 with $\chi(\mathrm{G})=3$, have equitable chromatic number at most 4 .

Proof. The result is a direct consequence of the analysis given in above subsection 3.1.

\subsection{Graphs $\mathrm{G}_{i}$ for which, $\chi\left(\mathrm{G}_{i}\right)=4$}

These graphs are $\mathrm{G}_{19}, \mathrm{G}_{20}, \mathrm{G}_{21}, \mathrm{G}_{22}, \mathrm{G}_{23}, \mathrm{G}_{24}, \mathrm{G}_{26}$ and $\mathrm{G}_{28}$.

(a) For graph $\mathrm{G}_{19}$, permissible Lucky partitions are,

$\left\{\left\{v_{1}, v_{3}\right\},\left\{v_{2}\right\},\left\{v_{4}, v_{6}\right\},\left\{v_{5}\right\}\right\},\left\{\left\{v_{1}, v_{5}\right\},\left\{v_{2}\right\},\left\{v_{3}\right\},\left\{v_{4}, v_{6}\right\}\right\}$.

Therefore, $\mathrm{L}_{\mathrm{G}_{19}}(6,4)=2 \lambda(\lambda-1)(\lambda-2)(\lambda-3)$.

(b) For graph $\mathrm{G}_{20}$, permissible Lucky partitions are,

$\left\{\left\{v_{1}, v_{6}\right\},\left\{v_{2}\right\},\left\{v_{3}, v_{5}\right\},\left\{v_{4}\right\}\right\},\left\{\left\{v_{1}\right\},\left\{v_{2}, v_{6}\right\},\left\{v_{3}, v_{5}\right\},\left\{v_{4}\right\}\right\},\left\{\left\{v_{1}\right\},\left\{v_{2}\right\},\left\{v_{3}, v_{5}\right\},\left\{v_{4}, v_{6}\right\}\right\}$.

Therefore, $\mathrm{L}_{\mathrm{G}_{20}}(6,4)=3 \lambda(\lambda-1)(\lambda-2)(\lambda-3)$.

(c) For graph $\mathrm{G}_{21}$, permissible Lucky partitions are,

$\left\{\left\{v_{1}, v_{5}\right\},\left\{v_{2}, v_{6}\right\},\left\{v_{3}\right\},\left\{v_{4}\right\}\right\},\left\{\left\{v_{1}, v_{6}\right\},\left\{v_{2}, v_{5}\right\},\left\{v_{3}\right\},\left\{v_{4}\right\}\right\}$.

Therefore, $\mathrm{L}_{\mathrm{G}_{21}}(6,4)=2 \lambda(\lambda-1)(\lambda-2)(\lambda-3)$.

(d) For graph $\mathrm{G}_{22}$, permissible Lucky partitions are,

$\left\{\left\{v_{1}, v_{4}\right\},\left\{v_{2}, v_{6}\right\},\left\{v_{3}\right\},\left\{v_{5}\right\}\right\},\left\{\left\{v_{1}, v_{5}\right\},\left\{v_{2}, v_{6}\right\},\left\{v_{3}\right\},\left\{v_{4}\right\}\right\},\left\{\left\{v_{1}, v_{5}\right\},\left\{v_{2}\right\},\left\{v_{3}\right\},\left\{v_{4}, v_{6}\right\}\right\}$.

Therefore, $\mathrm{L}_{\mathrm{G}_{22}}(6,4)=3 \lambda(\lambda-1)(\lambda-2)(\lambda-3)$. 
(e) For graph $\mathrm{G}_{23}$, permissible Lucky partitions are, $\left\{\left\{v_{1}, v_{4}\right\},\left\{v_{2}, v_{3}\right\},\left\{v_{5}\right\},\left\{v_{6}\right\}\right\},\left\{\left\{v_{1}\right\},\left\{v_{2}, v_{3}\right\},\left\{v_{4}, v_{5}\right\},\left\{v_{6}\right\}\right\},\left\{\left\{v_{1}, v_{4}\right\},\left\{v_{2}\right\},\left\{v_{3}, v_{6}\right\},\left\{v_{5}\right\}\right\},\left\{\left\{v_{1}\right\},\left\{v_{2}\right\},\left\{v_{3}, v_{6}\right\},\left\{v_{4}, v_{5}\right\}\right\}$. Therefore, $\mathrm{L}_{\mathrm{G}_{23}}(6,4)=4 \lambda(\lambda-1)(\lambda-2)(\lambda-3)$.

(f) For graph $\mathrm{G}_{24}$, permissible Lucky partitions are, $\left\{\left\{v_{1}, v_{5}\right\},\left\{v_{2}, v_{3}\right\},\left\{v_{4}\right\},\left\{v_{6}\right\}\right\},\left\{\left\{v_{1}, v_{6}\right\},\left\{v_{2}, v_{3}\right\},\left\{v_{4}\right\},\left\{v_{5}\right\}\right\},\left\{\left\{v_{1}, v_{5}\right\},\left\{v_{2}, v_{6}\right\},\left\{v_{3}\right\},\left\{v_{4}\right\}\right\},\left\{\left\{v_{1}, v_{6}\right\},\left\{v_{2}, v_{5}\right\},\left\{v_{3}\right\},\left\{v_{4}\right\}\right\}$. Therefore, $\mathrm{L}_{\mathrm{G}_{24}}(6,4)=4 \lambda(\lambda-1)(\lambda-2)(\lambda-3)$.

(g) For graph $\mathrm{G}_{26}$, permissible Lucky partitions are, $\left\{\left\{v_{1}, v_{5}\right\},\left\{v_{2}\right\},\left\{v_{3}, v_{6}\right\},\left\{v_{4}\right\}\right\},\left\{\left\{v_{1}, v_{5}\right\},\left\{v_{2}\right\},\left\{v_{3}\right\},\left\{v_{4}, v_{6}\right\}\right\},\left\{\left\{v_{1}\right\},\left\{v_{2}, v_{5}\right\},\left\{v_{3}, v_{6}\right\},\left\{v_{4}\right\}\right\},\left\{\left\{v_{1}\right\},\left\{v_{2}, v_{5}\right\},\left\{v_{3}\right\},\left\{v_{4}, v_{6}\right\}\right\}$, $\left\{\left\{v_{1}\right\},\left\{v_{2}\right\},\left\{v_{3}, v_{5}\right\},\left\{v_{4}, v_{6}\right\}\right\}$. Therefore, $\mathrm{L}_{\mathrm{G}_{26}}(6,4)=5 \lambda(\lambda-1)(\lambda-2)(\lambda-3)$.

(h) For graph $\mathrm{G}_{28}$, permissible Lucky partitions are, $\left\{\left\{v_{1}, v_{5}\right\},\left\{v_{2}, v_{3}\right\},\left\{v_{4}\right\},\left\{v_{6}\right\}\right\},\left\{\left\{v_{1}, v_{6}\right\},\left\{v_{2}, v_{3}\right\},\left\{v_{4}\right\},\left\{v_{5}\right\}\right\},\left\{\left\{v_{1}, v_{5}\right\},\left\{v_{2}, v_{6}\right\},\left\{v_{3}\right\},\left\{v_{4}\right\}\right\},\left\{\left\{v_{1}, v_{6}\right\},\left\{v_{2}\right\},\left\{v_{3}, v_{5}\right\},\left\{v_{4}\right\}\right\}$, $\left\{\left\{v_{1}\right\},\left\{v_{2}, v_{6}\right\},\left\{v_{3}, v_{5}\right\},\left\{v_{4}\right\}\right\}$.

Therefore, $\mathrm{L}_{\mathrm{G}_{28}}(6,4)=5 \lambda(\lambda-1)(\lambda-2)(\lambda-3)$.

Corollary 3.2. All graphs of order 6 and size 10 with $\chi(\mathrm{G})=4$, have equitable chromatic number equal to 4.

Proof. The result is a direct consequence of the analysis given in the above subsection 3.2.

\section{Conclusion}

It is important to note the difference between the Lucky partition numbers and the coefficient of the Lucky k-polynomial of a graph. For example, for $k=1, n \in \mathbb{N}$ all Lucky partition numbers equal 1. However, for all connected graph of order $n \geqslant 2$ the corresponding Lucky 1-polynomial coefficient is zero. Put differently, a proper 1 -colouring is not possible.

The procedure in Lemma 2.3 describes the construction of two intersection graphs from firstly, subgraphs of $G$ and secondly, from subgraphs of $\bar{G}$. The result in Theorem 2.4 and 2.5 can be viewed from the perspective of intersection graphs as well. Formalizing, results from an intersection graph perspective is a new avenue for research.

Much research has been done to gain a better understanding of the relations between the chromatic number and the independence number of graphs. There is a sense by the authors that research into the relations or bounds between the independence number and the perfect Lucky k-colourability are worthy avenues of research.

We observed in the introduction that the number of edges of $\bar{G}$ which are omitted from $E_{\chi}$ is the minimum number of bad edges in a bad chromatic completion of a graph G. A natural question arises, whether a similar result holds for bad k-colourings?

\section{References}

[1] J. A. Bondy, U. S. R. Murty, Graph Theory with Applications, American Elsevier Publishing Co., New York, (1976). 1

[2] D. Cvetković, M. Petrić, A Table of Connected Graphs on Six Vertices, Discrete Math., 50 (1984), 37-49. 3

[3] J. Kok, J. V. Kureethara, Stirling Numbers of the Fourth Kind E Lucky Partitions of a Finite Set, (Communicated). 2

[4] D. B. West, Introduction to Graph Theory, Prentice-Hall, Upper Saddle River, (1996). 1 